International Journal of Canadian Studies

Revue internationale d'études canadiennes

INTERNATIONAL JOURNAL OF CANADIAN STUDIES

REYUE INTERNATIONALE D'ÉTUDES CANADIENNES

\title{
" La nation québécoise et la crise des accommodements raisonnables: bilan et perspectives »
}

\section{Charles-Philippe Courtois}

Numéro 42, 2010

URI : https://id.erudit.org/iderudit/1002183ar

DOI : https://doi.org/10.7202/1002183ar

Aller au sommaire du numéro

\section{Éditeur(s)}

Conseil international d'études canadiennes

\section{ISSN}

1180-3991 (imprimé)

1923-5291 (numérique)

\section{Découvrir la revue}

Citer cet article

Courtois, C.-P. (2010). « La nation québécoise et la crise des accommodements raisonnables: bilan et perspectives ". International Journal of Canadian Studies / Revue internationale d'études canadiennes, (42), 283-306.

https://doi.org/10.7202/1002183ar

\section{Résumé de l'article}

La crise des accommodements raisonnables pose la question politique fondamentale de l'intégration de l'immigration au Québec. Cet émoi populaire déclenché par l'affaire du kirpan, met en relief une contradiction entre le multiculturalisme canadien et le modèle québécois. Le modèle canadien est conforme à une tradition de libéralisme pluraliste. Le modèle québécois, défini à partir de la Révolution tranquille et la loi 101, est davantage républicain: il insiste sur la liberté " positive ", sur l'intégration culturelle à la nation politique québécoise, avec la " convergence culturelle » et la démocratie québécoise a développé une certaine laïcité. Après avoir présenté ces deux modèles, puis distingué libéralisme et républicanisme, l'auteur examine les défis auxquels le modèle québécois est confronté: d'abord, sur le plan de la suprématie du modèle canadien en territoire québécois, conséquence de la Constitution de 1982 et de sa Charte; ensuite, au sujet de la francisation, dont les difficultés sont accrues à Montréal par le bilinguisme des services publics et parapublics, fédéraux et provinciaux. Face à ces défis, il recense les perspectives qui peuvent s'offrir à la nation québécoise, en deçà de la souveraineté, pour renforcer l'intégration au Québec et la cohérence du cadre civique québécois. Il s'agit essentiellement de renforcer la prédominance du français, notamment au niveau de la santé et des universités à Montréal, de sélectionner une immigration plus francotrope, et enfin d'établir une citoyenneté québécoise.
All Rights Reserved (C) Conseil international d'études canadiennes, 2010

Ce document est protégé par la loi sur le droit d'auteur. L'utilisation des services d'Érudit (y compris la reproduction) est assujettie à sa politique d'utilisation que vous pouvez consulter en ligne.

https://apropos.erudit.org/fr/usagers/politique-dutilisation/ 


\title{
Charles-Philippe Courtois
}

\section{"La nation québécoise et la crise des accommodements raisonnables: bilan et perspectives "}

\section{Résumé}

La crise des accommodements raisonnables pose la question politique fondamentale de l'intégration de l'immigration au Québec. Cet émoi populaire déclenché par l'affaire du kirpan, met en relief une contradiction entre le multiculturalisme canadien et le modèle québécois. Le modèle canadien est conforme à une tradition de libéralisme pluraliste. Le modèle québécois, défini à partir de la Révolution tranquille et la loi 101, est davantage républicain: il insiste sur la liberté "positive », sur l'intégration culturelle à la nation politique québécoise, avec la " convergence culturelle » et la démocratie québécoise a développé une certaine laïcité. Après avoir présenté ces deux modèles, puis distingué libéralisme et républicanisme, l'auteur examine les défis auxquels le modèle québécois est confronté: d'abord, sur le plan de la suprématie du modèle canadien en territoire québécois, conséquence de la Constitution de 1982 et de sa Charte; ensuite, au sujet de la francisation, dont les difficultés sont accrues à Montréal par le bilinguisme des services publics et parapublics, fédéraux et provinciaux. Face à ces défis, il recense les perspectives qui peuvent s'offrir à la nation québécoise, en deçà de la souveraineté, pour renforcer l'intégration au Québec et la cohérence du cadre civique québécois. Il s'agit essentiellement de renforcer la prédominance du français, notamment au niveau de la santé et des universités à Montréal, de sélectionner une immigration plus francotrope, et enfin d'établir une citoyenneté québécoise.

\begin{abstract}
The "crisis" surrounding reasonable accommodation poses the fundamental political question of immigration integration in Québec. The mass commotion set off by the kirpan case shines a light on a contradiction between Canadian multiculturalism and the Québécois model. The Canadian model conforms to a tradition of pluralist liberalism. The Québécois model, defined by The Quiet Revolution, notably with Bill 101, is more republican: it insists on "positive" liberty, on cultural integration with the Québécois political nation, on "cultural convergence", and Québécois democracy has developed a certain secularity. After presenting the two models and drawing the distinction of liberalism and republicanism, the author examines the challenges faced by the Québécois model; first, as regards the supremacy of the Canadian model on Québécois territory, a consequence of the 1982 Constitution and the Charter, and second, as regards Francization, which is increasingly difficult in Montréal with the bilingualism of public and parapublic services, both
\end{abstract}


federal and provincial. In the face of these challenges, the author enumerates the perspectives the Québécois nation might have-short of sovereignty-to reinforce integration in Québec and the coherence of the Québécois civic framework. Essentially, it comes down to reinforcing the predominance of French, notably at the level of health and universities in Montréal, selecting a more Francotrope immigration, and finally, establishing a Québécois citizenship.

La crise des accommodements raisonnables, qui a secoué le Québec de 2006 à 2008, pose une question politique fondamentale: celle de l'intégration de l'immigration au Québec. Qui définit les règles de l'intégration québécoise? Les institutions fédérales, en fonction d'une charte inscrite dans une constitution à laquelle le Québec n'a jamais adhéré, ou le Québec et les citoyens québécois? C'est aussi le résultat des frictions entre deux conceptions de la démocratie superposées sur un même territoire. D'emblée, nous écarterons la dichotomie entre ouverture et fermeture, laquelle nous apparaît faussée ici, même si beaucoup d'intellectuels et de journalistes ont voulu aborder ce débat sous cet angle ${ }^{1}$, pour le ramener aux questions politiques essentielles qu'il pose.

Précisons au préalable que nous parlons dans cet article de crise des accommodements raisonnables nonobstant l'affirmation du rapport Bouchard-Taylor selon laquelle il n'y aurait pas eu de crise. En fait, la crise dont nous parlons n'est pas une question liée au nombre d'accommodements jugés raisonnables ou déraisonnables, mais au fait avéré suivant: entre 2006 et 2008 un débat dans l'opinion publique s'est animé autour de cette question. $\mathrm{Au}$ fondement de cette crise, on trouve une critique dans l'opinion du modèle de gestion de la place de la religion dans l'espace publique et d'intégration associés aux « accommodements raisonnables » issus de la tradition juridique canadienne développée depuis 1982.

Ainsi, la question des accommodements raisonnables qui a soulevé un débat de grande ampleur dans l'opinion publique québécoise de 2006 à 2008 nous paraît poser une question de modèle d'intégration, mais aussi de modèle de démocratie (notamment au sujet de la place de la religion dans l'espace publique), alors que d'un côté des principes canadiens, articulés dans la charte de 1982, et de l'autre des principes québécois, exprimés dans plusieurs lois et politiques québécoises, mais aussi d'une certaine manière appuyés par l'opinion publique critique des accommodements raisonnables, se sont confrontés. Le jugement sur le kirpan est un exemple de cette confrontation. La commission Bouchard-Taylor, instituée par le gouvernement québécois, nous semble avoir proposé une voie de solution se rapprochant de l'intégration des normes canadiennes. Cela peut poser quelques problèmes que nous esquisserons en fin d'article. Auparavant, nous allons tâcher de caractériser 
les modèles canadien, plus libéral, et québécois, davantage républicain, en définissant d'abord ce que nous entendons par libéralisme et républicanisme.

\section{Libéralisme et républicanisme}

Quelle différence existe-t-il entre libéralisme et républicanisme? Il faut revenir sur l'histoire de la démocratie libérale moderne. Nous fondons notre réflexion sur des exemples concrets et historiques de République - plutôt que, par exemple, sur une idée kantienne du régime républicain².

La démocratie libérale est pour tous aujourd'hui un acquis si évident que, bien souvent, nous ne distinguons plus très bien ses deux composantes idéologiques, le libéralisme et la démocratie. Ces deux termes peuvent bien sûr renvoyer chacun à une certaine diversité, mais plus encore, leurs différentes combinaisons peuvent donner lieu à des régimes variés, comme la monarchie constitutionnelle britannique, la République américaine et la République française nous en offrent des exemples classiques. Dans certains cas, surgit une certaine opposition entre libéralisme et démocratie, mais aucun des trois régimes cités ne penche, depuis le XIX ${ }^{\mathrm{e}}$ siècle, exclusivement d'un côté. Il s'agit donc de différences de degrés dans la combinaison ou la hiérarchie des deux termes que nous allons tenter ici de dégager sur le plan des principes.

En outre, il faut souligner que d'aucuns englobent ces deux volets de la démocratie libérale dans le terme « libéralisme ». Louis-Georges Harvey utilise, à l'instar de Pocock, le terme " libéralisme constitutionnel » pour décrire le libéralisme distinct du républicanisme. Stéphane Kelly a distingué le libéralisme "Court Whig » du républicanisme anglais lié au "Country Party » du XVIII ${ }^{\mathrm{e}}$ siècle et à la première Révolution anglaise. On pourrait aussi décrire le libéralisme constitutionnel comme un libéralisme anglo-saxon, car il s'est déployé principalement dans des pays anglo-saxons (GrandeBretagne, Canada, Australie) et apparentés (Pays-Bas): voir Parenteau. Mais cela n'est pas exclusif ; c'est aussi, par exemple, le libéralisme de Benjamin Constant et de Guizot (Manent, 2002 et 2001; Constant; Renaut).

Enfin, pour les conservateurs « libéraux » au sens tory, c'est-à-dire des conservateurs qui acceptent le libéralisme constitutionnel (à la différence des réactionnaires ultras) il faudrait sans doute apporter d'autres nuances: ces derniers parleraient de « libéralisme progressiste » pour le libéralisme que nous allons décrire, le libéralisme conservateur accordant plus d'importance à l'héritage identitaire et culturel, dans la lignée de Burke. Néanmoins, ce libéralisme, tory aussi bien que whig, a été historiquement l'adversaire du principe de la souveraineté nationale et favorable au principe d'État multinational (impérial). C'était le cas dans l'Empire britannique au XIX ${ }^{\mathrm{e}}$ siècle et dans sa participation à la Sainte-Alliance; c'était le cas autant d'un whig comme Lord Durham (Bédard) que d'un tory comme John A. MacDonald. Le principe des nationalités, comme principe républicain, se voyait rejeté ; cela 
ne faisait pas pour autant davantage obstacle au patriotisme, ni non plus aux éventuelles expressions de chauvinisme britannique au sein de cet ensemble, que dans les pays où le principe national était affirmé.

Pour bien départager républicanisme et libéralisme, il faut identifier leurs valeurs prioritaires. Le libéralisme priorise la liberté individuelle définie par les intérêts et la propriété privée, liberté communément dite «négative», reliée à un idéal de progrès par le commerce. Le régime politique libéral idéal s'assure de garantir la liberté naturelle de l'individu. Et une constitution libérale, par la mixité des pouvoirs, ou la division des pouvoirs, s'assure de prémunir l'individu de tout abus de la part de l'État, en même temps que l'État garantit le respect de la propriété, des lois et du libre commerce. La communauté politique est d'abord un rassemblement de sujets de droit et la liberté individuelle est définie en ces termes. Cet idéal table sur une liberté privée accrue par la prospérité d'une société marchande, organisation laissant l'individu libre de vaquer à ses occupations.

Le républicanisme, en revanche, accorde la priorité au principe d'autodétermination du peuple, autrement dit de la souveraineté nationale, donc à la communauté politique nationale et au citoyen. Le bien commun y revêt une importance politique plus grande, puisque la communauté nationale y occupe une place presque entièrement occupée par le sujet de droit dans le libéralisme. C'est par sa participation civique à une nation libre que la personne est libre, c'est pourquoi on parle de liberté " positive». La définition de la liberté est donc plus politique; liée au libre arbitre ainsi qu'à une égalité de statut civique, elle est indissociable du statut de citoyen. L'égalité et la liberté républicaines se définissent en effet par le statut de citoyen de la nation, membre de la communauté politique. Dominique Schnapper a bien dégagé l'importance de la nation politique définie comme « la communauté des citoyens » dans le modèle républicain. Cet idéal table sur une liberté du citoyen fondée sur sa participation à la communauté politique nationale. Même dans son analyse nuançant l'importance du politique, et donc de la nation, dans sa définition de la liberté, en regard de la liberté privée, J.-F. Spitz fait ressortir le rôle accordé à l'État dans ce modèle, version moderne et plus libérale de la République des Anciens.

La Révolution américaine a proclamé le principe de la souveraineté du peuple. C'est une différence primordiale entre républicanisme et libéralisme. La Révolution française a été plus loin en précisant l'idée moderne de nation (Sieyès), qui est une idée politique, fondant la nation à la fois sur une unité culturelle et une volonté politique. Cette volonté, comme l'indique Renan, repose sur une mémoire et un vouloir vivre-ensemble à renouveler. Le printemps des peuples a poursuivi l'affirmation de ce principe. C'est le lieu ici de rappeler que le modèle de nation civique historique par excellence, le modèle français qu'on a coutume d'opposer au modèle ethnique allemand (Monière), 
n’a jamais été aculturel. Les études historiques sur la Révolution française rappellent d'ailleurs l'importance de la langue française dès la I ${ }^{\text {re }}$ République (de Certeau, Julia et Revel).

Ainsi, le républicanisme se démarque par l'importance qu'il accorde à la démocratie, à l'autodétermination, dans le diptyque « démocratie libérale ». En revanche, le libéralisme a pu, historiquement, se satisfaire de régimes non démocratiques - ce qui implique aussi qu'il accorde en principe moins d'importance civique à la nation (ce qui ne veut pourtant pas dire que le nationalisme y soit moindre). La monarchie constitutionnelle anglaise n'a pas toujours été démocratique. Au contraire, elle fut longtemps un régime mixte, de monarchie, d'aristocratie et de démocratie, et encore, une démocratie très fortement limitée par le suffrage censitaire. Il en reste des traces aujourd'hui dans des institutions comme la Chambre des Lords britanniques et le Sénat canadien, tous deux non élus, plus de deux siècles après les révolutions démocratiques des États-Unis et de France. Contrepartie logique, le républicanisme sera susceptible de limiter les droits individuels d'une manière qui pourrait apparaître inacceptable à certains tenants du libéralisme. Sans même parler de cas extrêmes et révolutionnaires - Robespierre n'a certainement pas toujours agit en démocrate - on pourrait simplement mentionner l'interdiction des symboles religieux ostentatoires appliquée aux écoles et aux fonctionnaires français.

Aujourd'hui, certains défenseurs d'une théorie des droits de l'homme fondée sur le droit naturel, cosmopolites, soutiennent une conception procédurale de la démocratie libérale qui tend à faire abstraction du peuple et de son autodétermination, donc de ce qui est proprement démocratique. Une conception procédurale du politique peut s'articuler à partir du sujet de droit du libéralisme. Une conception centrée sur les droits individuels nous paraît marquer les Commissaires Bouchard-Taylor et les experts qu'ils se sont adjoints, imprégnés de surcroît de la conception multiculturaliste de l'égalité différenciée. Citons par exemple cette phrase dans une section appuyant la notion juridique canadienne d'accommodement raisonnable: « Si on voulait caractériser en deux mots la notion d'accommodement, il faudrait dire: 1'égalité dans la différence », principe censé corriger le problème suivant:« Toute société a tendance à légiférer pour la majorité ; il s'ensuit que la loi n'est jamais vraiment neutre » (Bouchard-Taylor 63). C'est une égalité différenciée qui contraste avec l'égalité définie par le statut de citoyen des républicains, ce que traduit un aphorisme historique sur l'égalité laïque en France: « en tant que juifs vous n'avez aucun droit, mais en tant que Français vous les avez tous ».

Autre distinction: le libéralisme définit la neutralité en matière religieuse, principe fondamental des démocraties libérales, en fonction généralement d'un préjugé favorable à toutes les confessions. Historiquement, certaines étaient 
favorisées - comme les piliers historiques aux Pays-Bas et l'anglicanisme et le presbytérianisme au Royaume-Uni - mais la démocratisation des monarchies libérales les mène à définir ce préjugé favorable de façon égale, à l'instar du multiculturalisme canadien, mais aussi britannique et néerlandais. La République, en revanche, affirme le principe de laïcité (Rémond), comme établi ci-dessus. La neutralité de l'État en matière religieuse implique, en principe, de reléguer le religieux à la sphère privée et d'œuvrer à libérer la nation et le citoyen de l'emprise des Églises, l'ensemble s'appuyant sur un espace public (civique) laïque. Il s'agit d'une conséquence logique d'une définition politique de la liberté.

Voilàpourladéfinition destypesidéaux delibéralismeetderépublicanisme. Pour bien situer notre démarche, précisons que nous sommes redevables aux études remarquables de Louis-Georges Harvey et de Stéphane Kelly auxquelles nous devrions ajouter les réflexions de Marc Chevrier. Ces analyses nous ont aidé à préciser davantage les distinctions entre libéralisme et républicanisme. Notre analyse s'appuie aussi sur les travaux de chercheurs l'EHESS et de son centre Raymond-Aron ${ }^{3}$, sur la nation politique moderne et l'histoire de la démocratie, plus encore que sur les travaux de l'école de Cambridge (Pocock; Skinner). La littérature sur cette question est particulièrement marquée par les travaux fondateurs de Pocock, y compris chez Kelly, Harvey et Chevrier. Or, un modèle de démocratie libérale républicain par excellence est à notre sens le modèle républicain français, qui a développé davantage de distinctions avec le modèle libéral britannique que les États-Unis, à certains égards, notamment en matière religieuse. En contraste, bien qu'abordant l'évolution de la démocratie libérale dans l'Atlantique Nord, Pocock ne se penche pas sur le cas français pourtant déterminant, avec les cas anglais et américain, dans la révolution atlantique.

Deux chercheurs québécois, Harvey et Kelly, ont donc confirmé notre conviction de l'importance d'une tradition républicaine au Québec. Cependant, tout en avançant dans leur sillage, nous estimons pertinent de relever l'importance des influences non seulement américaines, mais aussi françaises, dans le républicanisme québécois, réalité mise en lumière dans un article récent (Courtois, 2009). Ainsi, en situant les États-Unis comme un modèle hybride entre libéralisme et républicanisme, tant sur le plan de la liberté, définie à la fois de manière libérale et républicaine, que de la neutralité en matière de religion, notre analyse se démarque quelque peu de la littérature qui s'appuie sur les travaux de Pocock. De fait, selon les termes de Stéphane Kelly, le parti fédéraliste américain (ce qui signifiait, comme au Canada, centralisateur), a promu une forte composante " Court Whig » dans le modèle états-unien. 


\section{Modèle québécois et modèle canadien}

On nous permettra de passer plus rapidement sur le modèle canadien, bien connu de par son aspect très officiel. Le Canada a développé une nouvelle politique d'immigration à partir des années 1960, alors qu'il mettait fin à la discrimination contre certaines origines, puis établissait, à partir des années 1970, une politique de multiculturalisme (1971), définissant le Canada comme une mosaïque sur le plan culturel et identitaire. La nouvelle Constitution de 1982, et la Charte des droits qu'elle enchâssait, font de ce multiculturalisme un principe actif: l'article 27 de la Charte en fait une clause interprétative. C'est donc dire que les institutions canadiennes encouragent désormais le maintien d'une certaine diversité culturelle parmi les nouveaux arrivants ou les minorités déjà établies au Canada (et non plus l'assimilation pure et simple au Canada anglais), voire le maintien d'une identité d'origine, au moins symboliquement, définissant largement la diversité culturelle sur une base ethnique - celle de l'origine. Ce modèle est fondé sur un libéralisme pluraliste déjà défendu par Pierre Elliott Trudeau dans Cité libre, et à présent par ce qu'on appelle couramment l'école canadienne de philosophie politique, en fait de libéralisme - les Will Kymlicka, James Tully, Charles Taylor et plusieurs autres penseurs de renom, se réclamant du libéralisme pluraliste (par ex. Maclure).

En revanche, le modèle québécois peut sembler un peu moins bien connu, parce qu'il demeure épars dans diverses législations et politiques dont la portée n'est pas aussi grande, dans notre système légal et politique, que celle de la Charte canadienne. Ce modèle s'est défini à la même époque que le nouveau modèle canadien, mais d'une façon quelque peu distincte. Ses orientations se définissent essentiellement par la conjonction de la Charte de la langue française et la politique de « convergence culturelle »: au lieu de favoriser la conception de la société comme mosaïque, le Québec favorise l'intégration à la communauté politique nationale, intégration à la fois culturelle et civique.

Le gouvernement québécois a voulu, à partir des années 1960, se réapproprier la compétence en immigration, reconnue par la Constitution de 1867 (article 95), en créant un ministère de l'Immigration (sous Daniel Johnson père ${ }^{4}$ ). Dans la foulée de l'affirmation nationale qui marquait le Québec avec la Révolution tranquille, tant sur le plan politique et gouvernemental que dans la société civile, le Québec s'est décidé à confronter la question de l'intégration culturelle de l'immigration québécoise. Cette intégration, jusqu'alors, se faisait largement au profit de la langue de la minorité anglophone dominante sur le plan économique ou, autrement dit, l'immigration québécoise s'intégrait directement à la majorité canadienne et non à la majorité québécoise. La volonté de s'approprier l'intégration de l'immigration au Québec, qui certes n'était pas neuve ${ }^{5}$, s'est affirmée avec la querelle des écoles de 
Saint-Léonard en 1968, puis s'est concrétisée avec la Charte de la langue française en 1977, qui elle-même répond à une longue série de requêtes en la matière (Jean-Claude Corbeil, pour la période post-1945), menées notamment par Armand Lavergne ou la Ligue des droits du français (Courtois, Trois mouvements intellectuels, 2007).

Que définit la Charte de la langue française en matière d'immigration? La loi 101 établit le français comme langue officielle du Québec, langue normale de travail et de l'école. De sorte que le français est appelé à être la langue de l'intégration, au travail aussi bien qu'à l'école. L'école est bien sûr déterminante pour la formation des citoyens. La Charte de la langue française affirmait ainsi clairement une volonté d'intégrer l'immigration à la nation, à la culture nationale commune du Québec, par la francisation, comme c'est courant dans les démocraties d'immigration. Relevons au passage que ces assimilations linguistiques se font automatiquement avec la naturalisation ailleurs - aux États-Unis, en France, mais aussi au Canada en quelque sorte, où l'anglicisation ne pose aucun défi, ou presque.

Cette politique établie par la loi 101 se voyait complétée par une série de définitions de la politique d'immigration du Québec (pour des résumés de cette politique, voir Labelle; Anctil). Dès 1971, le Premier ministre libéral du Québec, Robert Bourassa, signifiait le refus du Québec de souscrire à la politique du multiculturalisme (cité dans Paquette). Sous le gouvernement péquiste élu en 1976, se précisait une politique favorisant ce qu'on a appelé la « convergence culturelle», en d'autres termes l'intégration culturelle 6 . Ce principe était encore renforcé sous le second gouvernement de Robert Bourassa, avec l'accord-cadre Canada-Québec d3 1991 (Accord CanadaQuébec) et le contrat moral entre l'immigré et sa société d'accueil (Énoncé, 1990). Même en prenant le nom de politique d'interculturalisme, le principe était maintenu ${ }^{7}$; il a été réaffirmé en 2008 (Affirmer les valeurs communes).

Certes, dans une tentative un peu maladroite de normalisation avec le modèle canadien, Gérard Bouchard et Charles Taylor ont proposé en 2008 de redéfinir l'interculturalisme dans leur rapport, pour le rapprocher du multiculturalisme (Weinstock; Courtois, « Un rapport trudeauiste », 2008). Cette proposition ne paraît pas promise au succès, en regard de la nouvelle stratégie globale d'intégration adoptée par le gouvernement libéral en 2008, qui comporte un contrat moral, une " déclaration solennelle » par laquelle le candidat à l'immigration s'engage à respecter les " valeurs communes » du Québec, Charte de la langue française y compris (cité dans Baillargeon).

Avant d'aborder plus avant la question de l'interculturalisme, retenons que le principe d'intégration, dit de « convergence culturelle » comme nous l'avons relevé, maintenu dans la politique officielle (et malgré le rapport Bouchard-Taylor) est en accord avec l'esprit intégrateur de la loi 101. Le 
modèle québécois précise de surcroît un « devoir de participation civique », en accord avec la logique de liberté positive du républicanisme (Loi sur le ministère des Relations civiques et de l'Immigration du Québec, 1996, citée dans Labelle). Certes, les hésitations dans l'appellation du ministère des Relations civiques et de l'Immigration, ou des Communautés culturelles et de l'Immigration, correspondent non seulement à une alternance entre PQ et PLQ, mais à une oscillation dans la distance plus ou moins grande qui est prise avec le modèle canadien - ou l'assomption de cette distance. Néanmoins, en dépit de cette variation de terme, cet ensemble de politiques demeure cohérent.

Il faut enfin souligner que le Québec maîtrise la sélection des immigrants indépendants, ce qui constitue un peu moins de la moitié du total - le regroupement familial et les réfugiés complétant l'ensemble (Labelle) - et que le gouvernement fédéral « a seul la responsabilité des services relatifs à la citoyenneté » (Accord Canada-Québec 5, art. 28).

Outre ces limites, les différents éléments de la politique d'immigration du Québec passés en revue affirment clairement le principe d'une culture nationale commune à laquelle le nouveau citoyen québécois immigré est appelé à s'intégrer, à l'aide de la langue française. Voilà qui, en contraste avec le modèle canadien, exprime un principe plus républicain d'ouverture à la diversité ethnique. Notons d'ailleurs que dans son étude sur les modèles canadien, québécois et français de politiques d'immigration et d'intégration, Guillaume Rousseau situe le modèle québécois entre les modèles français et canadien, en d'autres mots, plus libéral que le français et plus républicain que le canadien. Des trois modèles, le multiculturalisme canadien affirme le principe le plus libéral d'ouverture à la diversité ethnique - mais aucun des trois n'est un modèle ethniciste fermé, ce qui est le propre d'États qui choisissent de limiter l'immigration et d'appliquer plus exclusivement le droit du sang à la citoyenneté (cela est le cas de démocraties libérales comme le Japon et l'Allemagne, jusqu'à un certain point, bien que le cas allemand soit en évolution).

\section{Intégration et républicanisme}

Nous l'avons signalé, dès la Ire République française, les principes du nationalisme civique défini alors avec le droit du sol et la fin de la discrimination religieuse ne faisaient nullement l'économie de la définition d'un contenu culturel de l'identité nationale, associée à la langue française. La culture seconde est ce qui constitue la culture nationale commune ; elle s'acquiert par l'instruction et ne dépend pas de critères de naissance. En matière d'unité culturelle, nous faisons donc référence à la notion de culture seconde exposée par Fernand Dumont (voir aussi Thériault). Ce n'est que dans des débats très récents qu'on a défendu un modèle de nation civique qui serait aculturel et 
tenté de qualifier d'ethniques les éléments de contenu culturel (Bock-Côté ${ }^{8}$ ). On pourrait dire que, à ce moment, la distinction entre nationalisme civique et patriotisme constitutionnel a été brouillée.

Il en va d'ailleurs de même pour la prétendue « laïcité ouverte », terme novateur défendu soudain au Québec par des partisans des principes du multiculturalisme canadien (Bouchard et Taylor; Leroux; Maclure) ; elle ne correspond à aucun modèle historique concret, sinon aux modèles de libéralisme anglo-saxons décrits ci-dessus. La défense de la laïcité au Québec, avant que le tout récent terme de laïcité ouverte ne soit forgé, s'inspire de l'exemple historique connu, la République française. La laïcité est en effet un concept issu de la République française ; la République américaine comme le régime britannique ont développé des concepts de neutralité en matière religieuse, mais pas de laïcité. Or, la « laïcité ouverte » n'est qu'une nouvelle manière de nommer les principes énoncés dans les politiques de multiculturalisme des monarchies constitutionnelles britanniques. Bref, tout comme le nouveau nationalisme civique, lorsque confondu avec du patriotisme constitutionnel, n'est plus du nationalisme, la « laïcité ouverte » n'a pas grandchose à voir avec la laïcité.

À ce sujet, notons que la République des États-Unis, sans pour autant verser dans le multiculturalisme, apparaît effectivement comme un modèle hybride de libéralisme et de républicanisme, puisque, entre la laïcité à la française et la neutralité religieuse du libéralisme constitutionnel, elle est conforme au second modèle. En comparaison des Pays-Bas et du Royaume-Uni, dont les États-Unis héritent sur ce plan, ils ont simplement affirmé de manière plus achevée le principe de neutralité, en n'accordant pas de privilège à des Églises particulières.

Pour revenir à l'importance de l'unité culturelle dans la nation civique (Courtois, " Histoire, identité, démocratie », 2008), Pierre Rosanvallon, à travers ses nombreuses études sur la démocratie moderne, a rappelé l'importance de la nationalité', autrement dit de la frontière, dans l'existence d'une communauté politique ; l'étranger est défini en termes politiques mais l'intégration à la communauté politique, qui est ouverte, appelle une certaine forme d'assimilation. Yves Lacoste, géopolitologue, a quant à lui plaidé pour la revalorisation de la nation comme communauté politique et siège de l'autodétermination, mais aussi pour la reconnaissance de ce fait: en pratique, la nation civique à la française repose sur une unité culturelle mais non ethnique. C'est bien la logique défendue par la loi 101 dans son esprit.

Lorsqu'on se reporte au célèbre texte de Renan, Qu'est-ce qu'une nation?, on constate qu'il fait justement ce type de distinction, quoique de manière moins claire et aboutie. Renan soutient que la langue et l'ethnie ne sont pas déterminantes dans la constitution d'une nation, communauté volontaire. 
Cependant, il insiste sur la nécessaire mémoire partagée, critère qui renvoie à une forme d'identité commune. Nous pourrions avancer que cela rappelle l'adhésion à une culture seconde que l'école républicaine pourvoie et qui constitue la culture commune. Dans le cas cité par Renan, l'Alsace-Lorraine, rappelons que la variété de langues maternelles qui existe en France (dialectes romans et langues régionales de souche non gallo-romaine en périphérie) n'empêche pas une culture commune de s'exprimer à travers une langue nationale, le français, y compris au XIX ${ }^{\mathrm{e}}$ siècle, en France. Cette culture nationale (seconde) est distincte de la culture familiale et ethnique (première) bien que normalement elle en constitue le développement sur le plan de la haute culture - comme c'est le cas en France entre les patois de langue d'oïl et le français standard - même s'il y a des exceptions, comme l'alsacien. La culture nationale (seconde) est aussi un espace partiellement neutralisé sur certains plans, comme l'implique le principe de laïcité (qui n'efface pourtant pas des héritages catholiques de la culture commune française).

On comprend, en conséquence, l'importance de l'école républicaine pour former tous les citoyens de la nation, natifs ou adoptés. L'école donne la maîtrise de la culture seconde nationale. Encore une fois, on peut constater que c'est bien la logique que promulgue la Charte de la langue française.

Ces distinctions posées, le modèle québécois, tel que développé depuis la Révolution tranquille, se révèle davantage républicain tandis que le modèle canadien s'avère libéral et " court whig ». Au vrai, comme le multiculturalisme canadien se distingue du multiculturalisme " structurel » (Quérin 9), adopté par exemple aux Pays-Bas, il maintient une certaine intégration. Seulement, la loi sur le multiculturalisme et la clause interprétative de la Charte en font un principe actif par lequel les institutions canadiennes encouragent le maintien d'identités ethniques issues de l'immigration. Le Québec, en revanche, encourage la convergence culturelle et la participation civique à la nation québécoise. Il faut dire que l'anglicisation et l'intégration à la culture majoritaire qu'elle implique, ne posent guère de problèmes au Canada anglais, en tout cas en regard des défis de la francisation et de l'intégration à la culture majoritaire québécoise. On relève cependant que de plus en plus de propositions sont mises de l'avant qui prétendent que les principes du multiculturalisme canadien vont exiger le développement d'un multiculturalisme structurel; on songe au débat sur la charia ontarienne et à l'instauration d'écoles pour les Noirs à Toronto.

Il est pertinent d'analyser l'interculturalisme québécois en relation avec la culture nationale commune du modèle républicain. La politique d'interculturalisme peut être interprétée comme encourageant l'échange interculturel au niveau de la culture première et la convergence culturelle au niveau de la culture seconde, spécialement par l'école. C'est l'interprétation que nous soutenons, à l'instar notamment de J.-Y. Thériault. Si Guillaume 
Rousseau a raison de classer le modèle québécois comme intermédiaire entre le canadien et le français, c'est à cause des ouvertures au pluralisme inscrites dans l'interculturalisme et la Charte des droits et libertés de la personne du Québec. Or, l'article 43 de la Charte québécoise, qui nomme le droit aux associations et manifestations culturelles minoritaires, n'est pas comparable au principe activiste du multiculturalisme canadien. En effet, l'article 43 ne constitue pas une clause interprétative, mais simplement un droit social sans préséance sur les lois ordinaires (Charte québécoise des droits et libertés, art. 52). À ce titre, il serait sans doute possible de le comparer à une simple liberté d'association, laquelle s'insère parfaitement dans une logique républicaine. Au vrai, l'interprétation devient ici déterminante, et dépend des autres lois fondamentales en regard duquel cet article sera compris. S'il était analysé en fonction de la Charte de la langue française et d'une loi sur la convergence culturelle (qui n'est qu'une politique), ce serait autre chose que lorsque la Charte des droits et libertés québécoise est interprétée en fonction de la Charte des droits canadienne ${ }^{10}$, sa puînée. La question de l'interprétation est centrale bien sûr, d'ailleurs la Cour d'appel du Québec et la Cour suprême du Canada n'ont pas eu la même interprétation de la liberté de religion dans les jugements sur le kirpan. Ces interprétations sont marquées par des modèles différents.

En somme, le modèle québécois accorde une importance politique et culturelle à la nation dans ses principes d'intégration. La Révolution tranquille, époque d'affirmation politique nationale, s'est accompagnée d'une prise en charge par l'État québécois de responsabilités dévolues à l'Église. La laïcisation qui s'est alors accomplie s'est faite dans l'affirmation graduelle du principe de laïcité - et pas seulement de neutralité à l'anglo-saxonne. La laïcisation des commissions scolaires il y a une dizaine d'années consacrait ce principe. Le Québec s'est aussi doté d'une «Assemblée nationale », appellation à connotation républicaine, sous la gouverne de Johnson père. On aura compris qu'une démocratie peut adopter un caractère plus républicain sans être un régime républicain au sens strict, c'est-à-dire sans monarque, comme l'exprimait à sa façon Jean-Jacques Rousseau en 1762 lorsqu'il définit la République comme, en pratique, tout régime où le peuple est le véritable souverain. De fait, le modèle québécois met l'accent sur l'intégration à la culture nationale commune et donc à la nation québécoise, communauté politique unie par une langue, une mémoire, une volonté, comme l'expriment la loi 101 et la politique de convergence culturelle.

\section{La légitimité de l'intégration}

Reste un dernier point à préciser avant d'aborder les défis de la nation québécoise en matière d'intégration: la légitimité du principe d'intégration de l'immigration lui-même, ou, autrement dit, le bien-fondé de l'intégration culturelle. Dans le cas de penseurs libéraux, l'intégration nationale, soit 
l'assimilation de l'immigration, paraît jusqu'à un certain point illégitime, c'est-à-dire dès qu'on invoque des principes contraignants et tranchés, aussi tranchés en matière culturelle que la laïcité peut l'être en matière religieuse. « Illibérale » pour Ray Taras et injuste, jusqu'à un certain point, pour Kymlicka $(1989 ; 1995)$ qui accepte certes les normes d'intégration (et il reconnaît le principe des deux sociétés d'accueil au Canada). Cependant, l'assimilation peut constituer à ses yeux une injustice s'il s'agit d'une démarche plus contraignante qu'une invitation, et avec un contenu culturel plus fortement affirmé que certaines normes libérales, égalitaires et démocratiques - songeons aux exemptions à la loi qui doivent être dessinées, selon lui, pour accommoder les minorités religieuses, par exemple. Cela découle d'une pensée de la liberté centrée avant tout sur le sujet de droit. Kymlicka se penche ainsi sur les revendications individuelles, et en l'occurrence celles de minoritaires, pour définir ce qui est juste. Celles-ci peuvent être promues par des regroupements ethniques ou ethno-religieux qu'il s'agira de reconnaître, et en ce sens Kymlicka appuie une forme d'égalité différenciée et de référence à la croyance « authentique » (fondement de la notion canadienne d'accommodement raisonnable).

Un nouvel exemple de cette logique nous est offert par le jugement de la Cour suprême sur la loi 104, rendu le jeudi 22 octobre 2009, qui invalide la loi en affirmant que les mesures du législateur québécois seraient « draconiennes » et même « excessives », et qu'il devrait s'efforcer d'examiner au cas par cas «l'authenticité de l'engagement des parents à cheminer dans la langue de la minorité » (jugement cité dans Robert Dutrisac, 2009).

En revanche, dans une logique républicaine, cette question est à aborder en premier lieu en fonction de l'autodétermination d'un peuple, fondement incontournable de la démocratie. Pour être politiquement libre, il faut être membre d'une nation libre. Or, pour être souverain, mais aussi pour être autonome (cas du Québec), un peuple doit par définition maîtriser les flux migratoires sur son territoire.

Pierre Manent (2006) a dernièrement rappelé « la raison des nations » et donc de la frontière en démocratie. S'il ne pouvait contrôler son territoire devant un déplacement massif de population issue d'un pays beaucoup plus peuplé, un peuple perdrait alors toute autonomie, réalité qui se produit au Tibet, par exemple. Par conséquent, toujours en fonction de ce principe premier de la liberté démocratique qu'est le principe des nationalités, il apparaît foncièrement légitime qu'une nation choisisse la politique l'immigration qui lui sied et qui lui plaît. Certaines choisiront de n'avoir pas, ou très peu d'immigration, malgré une démographie déclinante tels le Japon ou la Norvège. D'autres adopteront des modèles multiculturels - comme les Pays-Bas, le Canada, le Royaume-Uni. D'autres encore choisiront l'intégration républicaine et le melting pot comme la France et les États-Unis. Et de fait, il est question ici de 
la défense du principe du creuset québécois. Il faut avouer, du reste, que ces modèles achevés, français et états-unien, n'imposent pas d'assimilation sur le plan de la culture première mais bien de la culture seconde (Taguieff $\left.{ }^{11}\right)$, et que c'est aussi la voie empruntée par le modèle québécois.

En outre, l'assimilation ne nous semble répréhensible que dans le cas de l'annexion d'un peuple, et non dans le cas de l'adhésion d'un sujet libre à une nouvelle nation dont il choisit de devenir citoyen à travers un processus migratoire. Personne n'oblige le candidat à l'immigration à s'établir chez cette nation et à en demander la naturalisation s'il ne désire point cette assimilation. Du reste, l'assimilation est non seulement légitime en matière d'immigration mais son terme naturel. Par la force des choses, la descendance de l'immigré, si son intégration est réussie, au fil des générations, se fondra dans le tout de la nation d'accueil. Toujours en fonction de ses intérêts nationaux, il nous appert parfaitement légitime qu'une nation autonome choisisse de favoriser l'assimilation de son immigration.

$\mathrm{Au}$ surplus, à la différence de toute une tradition multiculturaliste développée par l'État canadien et par l'école de pensée politique canadienne, entre autres, nous ne sommes pas convaincus que la création de « communautés culturelles » concrétise véritablement la demande des individus immigrés. Par exemple, la question des tribunaux de la charia en Ontario relevait-elle des demandes de la majorité des musulmans ontariens ou d'une minorité d'intégristes? Mais plus fondamentalement, le principe même de démocratie nous semble exiger la reconnaissance du droit des nations à fixer les règles de leur politique d'immigration, qui n'est jamais un droit universel, mais toujours un privilège offert à certaines conditions. La question ne nous apparaît donc pas devoir être abordée en fonction des préférences de chaque individu pris isolément mais en fonction des conditions de la vie commune de la Cité et de la volonté d'un peuple.

\section{Les problèmes concrets du Québec en matière d'intégration}

Après cette présentation du modèle québécois, examinons maintenant les difficultés rencontrées par son application sur le territoire québécois. En premier lieu, le modèle québécois rencontre une difficulté en matière de cohérence, parce qu'il entre légalement en conflit avec le modèle canadien qui s'applique simultanément sur son territoire. En second lieu, il rencontre des défis culturels et linguistiques singuliers, liés également à sa situation politique. Ces deux phénomènes se conjuguent et posent ensemble un défi de taille à la perpétuation de la nation québécoise. C'est pourquoi Guy Laforest soutient que le multiculturalisme « est quelque chose que le Québec ne peut se permettre » (53). Laforest rappelle d'ailleurs que le rapport Allaire avançait déjà que « Le maintien du multiculturalisme au Canada joue contre la population francophone » (cité dans Laforest 49). Ce rapport, certes émis en période 
de crise politique, exprimait une forme de consensus dépassant largement les souverainistes québécois puisqu'il émanait du PLQ et rejoignait à cet égard une critique du multiculturalisme exprimée par le Premier ministre libéral Robert Bourassa dès le début des années 1970.

Ainsi, en mars 2006, la Cour suprême du Canada renversait le jugement de la Cour d'appel du Québec et invalidait la décision d'une commission scolaire de Montréal d'interdire le port du kirpan ${ }^{12}$, couteau religieux sikh, dans ses écoles au même titre que les autres armes blanches. Plus précisément, le Conseil d'établissement de l'école avait critiqué en 2002 une proposition d'accommodement raisonnable (ainsi que le Procureur général du Québec), suggérant de plutôt admettre le port d'un kirpan fait d'un matériau inoffensif, ce que la Cour d'appel du Québec avait appuyé par son jugement, en rappelant que la liberté de conscience religieuse doit se conformer au respect des valeurs démocratiques du Québec (Commission scolaire Marguerite-Bourgeoys c. Multani §72-74), et que l'impératif de sécurité n'était pas moindre dans une école que dans une cour ou un avion (\$84). La Cour suprême renversa cette décision au nom de la Charte canadienne enchâssée dans la Constitution de 1982, mais aussi au nom de la Charte québécoise interprétée à la lumière de la première (Multani c. Commission scolaire Marguerite-Bourgeoys). Il faut se rappeler d'abord que le Québec n'a pas adhéré à cette Constitution. Ensuite, que le principe du multiculturalisme qui y est consacré se veut actif, interprétatif. La Cour suprême veut donc que la manifestation d'identités issues de l'immigration et minoritaires obtienne une forme de reconnaissance officielle. C'est la raison essentielle de cette décision, qui ne répond pas à la question de sécurité posée par la Commission scolaire dans une conception plus laïque et républicaine de l'égalité de tous devant la loi (non-différenciée). Car en matière de sécurité, le port du kirpan est interdit à l'intérieur de la Cour suprême ellemême ! D'autre part un incident dans une école de l'arrondissement Lasalle à Montréal a rappelé la possibilité de contentieux en cette matière dans les écoles ${ }^{13}$.

Par cette décision, la Cour suprême illustrait le clivage entre le modèle québécois et le modèle canadien, en particulier à propos de la neutralité en matière de religion. Elle le fit bien sûr en imposant le modèle canadien, ce qui suscita un tollé dans l'opinion. Or, depuis 1982, la Cour suprême a aussi invalidé de nombreux aspects de la loi 101, ce qui met à mal le renforcement de la culture nationale commune au Québec. Par toutes ces décisions, elle révèle les difficultés de la nation québécoise à faire valoir ses choix démocratiques collectifs sur son propre territoire, à s'autodéterminer en somme, même dans des domaines de compétence provinciale, et même si elle rejette la Constitution de 1982. Bien entendu, l'opinion québécoise n'est pas attachée à l'idée républicaine en tant que telle, mais plus directement aux intentions annoncées tout d'abord dans le modèle québécois tel qu'il avait commencé à être défini par la loi 101, dans une logique républicaine, en faveur de l'intégration 
culturelle. C'est ce principe fondamental, soit que la communauté politique nationale québécoise puisse définir les normes de l'intégration en fonction de ses intérêts (la francisation) et de ses préférences (la laïcité), que favorisait l'opinion presque unanime en défaveur de la décision de la Cour suprême sur le kirpan.

Par ailleurs, les problèmes d'intégration au Québec demeurent concrètement nombreux, malgré les améliorations apportées par la loi 101, le développement d'une politique d'intégration, et les succès de l'affirmation nationale en général - par exemple dans le domaine économique. Les résultats sont probants: d'environ $80 \%$ d'immigrés s'anglicisant avant la loi 101 on est passé à environ 50\% (Paillé14). C'est une amélioration importante mais non satisfaisante. L'anglicisation au Canada anglais atteint des taux bien supérieurs, et bien sûr sans partage avec une autre société d'accueil ${ }^{15}$.

Plusieurs facteurs minent l'intégration au Québec. La loi 101 ne s'applique pas aux compagnies à charte fédérale comme plusieurs banques, ni aux compagnies fédérales. Ces milieux, comme on peut le voir en prenant le train ou l'avion à Montréal, sont fortement anglicisants, sur le plan de la langue commune (de travail comme de relation avec les clients). L'État canadien envoie au nouveau citoyen québécois un autre message, celui d'un pays bilingue et multiculturel ; alors que l'anglais prime sur notre continent, il prime aussi au Québec en contexte de bilinguisme officiel. Ce message, qui relativise fortement toute incitation à l'intégration en français et à la majorité québécoise, est consacré par la naturalisation canadienne.

À cela il faut ajouter des services sociaux largement bilingues à Montréal bien que de compétence provinciale. Ainsi, le gouvernement québécois ne respecte plus la logique de ses propres lois, notamment de la loi 101. Une série d'articles du journaliste Robert Dutrisac (2008) l'a récemment démontré de façon accablante. Depuis le refus du gouvernement Bouchard de remettre en question la loi 178 écrite en réponse à une invalidation de la Cour suprême, les partis politiques québécois au pouvoir, péquistes comme libéraux, ont cédé à une conception libérale et canadienne, voire trudeauiste, du Québec, comme État bilingue répondant à un droit individuel de bilinguisme. L'intégration par les services de la fonction publique québécoise, ne se fait donc pas véritablement en français, malgré le message officiel du gouvernement.

De surcroît, la part de financement recueillie par les établissements d'enseignement supérieur et les établissements de santé anglophones, au Québec, est bien supérieure à la proportion des anglophones de naissance (qui est de $8 \%$ ) ou d'usage $(10 \%)$. Plus de la moitié des professeurs d'université, à Montréal, travaillent dans les deux universités anglophones de la ville (Chevrier). Avec environ 20\% d'anglophones dans la région 
métropolitaine, c'est pourtant une bonne moitié du système de santé de la métropole qui est anglophone. Le plus gros Cégep du Québec est anglophone et il peut être difficile, en pratique, d'obtenir des soins de santé en français à Montréal. Même en concédant pro forma que la majorité des établissements de santé rattachés à McGill offrent un bilinguisme impeccable, ce qui est loin d'être prouvé, force est de reconnaître que ces institutions sont des lieux de travail anglophones, et donc anglicisateurs pour les immigrants (et les francophones) qui y sont intégrés. Comme se concentrent à Montréal à la fois les institutions de la minorité anglaise et l'établissement de $90 \%$ des immigrés - encore $88 \%$ des allophones habitaient la région montréalaise en 2001 (Paillé 9) - cette faiblesse des institutions françaises dans la métropole implique des difficultés multiples pour la perpétuation nationale. Bref, la nation québécoise, tout en faisant le choix d'une politique d'immigration, fait face à de sérieux et d'exceptionnels défis d'intégration.

\section{Les perspectives}

Il est par conséquent possible d'affirmer que la crise des accommodements raisonnables exprimait la prise de conscience d'une difficulté dans l'application du modèle québécois, qui entre en contradiction avec le modèle canadien sur le territoire québécois, et une volonté de régler ce problème. Or le rapport Bouchard-Taylor a préconisé ce que nous appellerions une harmonisation du modèle québécois avec les principes canadiens. Notamment, en critiquant le principe de convergence culturelle pour lui préférer ce que les auteurs appellent une « culture citoyenne » (Bouchard-Taylor 125). Aux yeux des commissaires, des critiques émises dans les années 1990 l'auraient rendue dépassée: « La culture publique commune faisait [alors] l'objet de critiques ; on lui reprochait notamment de trop orienter la dynamique interculturelle vers l'assimilation aux traditions judéo-chrétiennes et à la culture francophone. On avait fait un procès analogue à la notion de culture de convergence, que l'on estimait trop axée sur la culture "française" (Bouchard-Taylor 117).

En ce sens, le rapport Bouchard-Taylor, en proposant simplement de redéfinir le modèle québécois en fonction de principes de laïcité ouverte et de patriotisme constitutionnel - sans l'affirmation d'une culture de convergence - davantage conforme à la jeune tradition de multiculturalisme canadien, nous semble d'une part avoir simplement repoussé à plus tard la résolution de ce conflit lancinant entre deux modèles. Mais aussi, d'autre part, le rapport ne semble pas avoir proposé de solution adéquate du point de vue du souci d'intégration nationale au sens fort exprimé alors. Il demeure un véritable défi pour la perpétuation de la nation québécoise mais aussi, pourrait-on avancer, pour la démocratie québécoise, alors que l'opinion incline à préférer une autre voie que celle tracée par la Cour suprême et la tradition canadienne. 
Devant les pressantes difficultés que nous avons tâché de passer en revue, ceux qui, et ils sont nombreux, se soucient de l'avenir de la nation québécoise et de la perpétuation de son identité, demanderont: " Que faire? » Quelles perspectives s'offrent à la nation québécoise pour améliorer l'efficacité de son intégration et la cohérence dans l'application de ses choix collectifs, en deçà de la souveraineté?

Certes, faire de l'État québécois un État pleinement souverain, c'est-àdire indépendant, offrirait la possibilité de régler ces aspects de l'intégration, en respectant de manière cohérente les principes de la prédominance du français et la convergence culturelle comme de la laïcité. Mais le Québec ne peut rester les bras croisés dans l'attente de cette souveraineté, qui, au reste, n'est pas à l'horizon politique à moyen terme. Il serait plus sage d'agir promptement, comme il l'a déjà fait par le passé, notamment avec la loi 101.

Les propositions de solutions sont nombreuses. Christian Dufour plaide, à notre avis avec beaucoup de réalisme, pour la réaffirmation nette et cohérente du principe de claire prédominance du français. Il s'agit au premier chef de l'État québécois et des lois québécoises. Mais ne faudrait-il pas l'exiger aussi des compagnies à charte fédérale et des compagnies fédérales au Québec, comme les ports, les aéroports et les compagnies de transport? Le Bloc québécois quant à lui a proposé au printemps 2008 d'élargir son application aux institutions relevant du fédéral (projet de loi C-482) et de soustraire le Québec à la loi sur le multiculturalisme (projet de loi C-505). Voilà deux mesures qui seraient significatives. Mais est-il réaliste de croire que le gouvernement fédéral pourrait les appuyer? Il faudra voir si la proposition du NPD faite à l'automne 2009 (projet de loi C-455) en matière de langue aura plus d'appétence pour le gouvernement fédéral - ou une autre du même type, à terme.

Il faudrait cependant que l'État provincial québécois renoue lui-même avec la cohérence en cette matière - et donc les partis qui le gouvernent - en appliquant réellement l'esprit de la loi 101 aux services de la fonction publique. Comme l'a récemment souligné Marc Chevrier, il faudra également confronter ce problème sur le plan des services des établissements de santé et postsecondaires, où il se pose avec une acuité particulière à Montréal. Chevrier suggère de créer une nouvelle faculté de médecine au sein du réseau de l'Université du Québec, ce qui aurait l'avantage d'aider le français à Montréal dans les deux secteurs clés de la santé et de l'université.

Par souci d'équité, et parce que c'est une question vitale pour l'intégration et donc la pérennité de la nation, ne faudrait-il pas faire en sorte que les universités francophones représentent plus que $50 \%$ de l'offre et obtiennent plus que $50 \%$ des budgets, et que les établissements francophones de santé à Montréal représentent 60 ou $66 \%$ de l'offre et de l'activité? S'offre également 
la solution d'étendre les dispositions scolaires de la loi 101 aux Cégeps, que défend le démographe Marc Termote de l'IRFA (Institut de recherche sur le français en Amérique, cité dans Gervais).

Une autre série de mesures pourrait viser à franciser davantage l'immigration à la source. Il s'agirait d'augmenter la proportion de « francotropes »- ressortissants des pays de langue latine et de la francophonie, comme des candidats d'autres pays connaissant déjà le français ou l'apprenant avant d'arriver - ou encore d'exiger une connaissance minimale du français (Dubreuil, 2006; 2007). C'est sans doute plus efficace que l'extension des dispositions de la loi 101 en matière de langue de travail à toutes les PME de moins de 50 employés ${ }^{16}$ - mesure à envisager par ailleurs.

Enfin, il reste un élément clé de toute politique d'immigration et d'intégration: la naturalisation. Une remise sur le métier du projet de loi 195 serait donc pertinente. Pour instaurer pareille citoyenneté québécoise, il faudrait en premier lieu l'accorder à tous les citoyens canadiens résidants du Québec. Ensuite, il faudrait rendre obligatoire l'obtention de cette citoyenneté par tous les immigrants au Québec, qu'ils relèvent directement des flux de population maîtrisés par le fédéral ou des catégories sélectionnées par le Québec. Un tel processus rendrait les cours de connaissance minimale du Québec et du français obligatoire pour tous, comme la naturalisation le fait ailleurs, comme d'ailleurs elle le fait pour le Canada. Il faudrait sûrement restaurer la structure d'accueil des COFI (Centres d'orientation et de formation des immigrants).

Mais pourquoi ne pas aller plus loin, et réclamer la compétence du Québec - reconnu comme nation - en matière de naturalisation, dans un nouvel accord-cadre? En somme, au lieu de créer un processus parallèle de citoyenneté, il faudrait obtenir que le Canada reconnaisse pour le Québec la compétence en matière de droit de cité, comme en Suisse. Dans la confédération helvétique, en effet, ce sont les Cantons qui octroient le droit de cité ${ }^{17}$ (le fédéral décernant une autorisation). Ce qui veut dire que quiconque immigre dans le Canton de Genève, se voit accorder par Genève la naturalisation à titre de citoyen du Canton, et du coup de citoyen suisse avec le passeport qui l'accompagne. Si cette compétence est d'importance pour un Canton suisse, à plus forte raison est-elle de rigueur pour le Québec. Sa situation géographique et politique, combinée au choix de l'immigration pour répondre à des carences de natalité, en font un élément crucial pour l'avenir de la nation québécoise. 


\section{Notes}

1. À titre d'exemple, le chroniqueur Vincent Marissal écrivait que l'expression populaire à la Commission Bouchard-Taylor ne faisaient pas chic « devant la visite » (dans « Avant d'enterre la coalition », La Presse, 14 sept. 2007 ; voir aussi sa chronique « Le freak show », La Presse, 13 sept. 2007), tandis que le politologue Guy Lachapelle, incapable de soutenir le spectacle de cette expression, en appelait à la cessation des travaux de la commission : « Assez, c'est assez! », Le Devoir, 5 nov. 2007. Voir à ce sujet Joseph Facal, « Les vierges offensées », Le Journal de Montréal, 7 nov. 2007.

2. À la différence de certains théoriciens, comme Juliette Grange dans L'Idée de République, Paris, Poket, 2008.

3. L'EHESS et son centre de recherches politiques Raymond-Aron et accueillent ou ont accueilli feu François Furet, feu Michel de Certeau, Marcel Gauchet, Pierre Manent, Mona Ozouf, Pierre Rosanvallon, Dominique Schnapper.

4. En 1968. Dès 1965, suite à une motion du député unioniste Gabriel Loubier, le gouvernement Lesage avait créé un service québécois d'immigration. Référence : Bilan du siècle, 1 [sic] mars 1965. http://bilan.usherbrooke.ca/bilan/pages/evenements/20224.html

5. On peut citer les initiatives de l'époque où Hector Fabre était agent du Québec à Paris, puis quand Olivar Asselin fut nommé commissaire à l'immigration, au tout début $\mathrm{du} \mathrm{XX}^{\mathrm{e}}$ siècle, ou encore les propositions d'un publiciste républicain comme Wilfrid Gascon qui, dans l'enquête Notre avenir politique tenue par l'Action française, préconise le développement d'une immigration « assimilable », tout comme la contribution de Bruchard à cette même enquête dénote cette volonté d'intégration au sein du mouvement national. L'Action française [Lionel Groulx (dir.)], Notre avenir politique, Montréal, Bibliothèque de l'Action française, 1923.

6. « ... le bien commun et l'intérêt même des minorités exigent que ces divers groupes s'intègrent à un ensemble québécois essentiellement francophone », QUÉBEC, Comité ministériel permanent du développement culturel, 1978, p. 63.

7. «En 1978, le gouvernement introduisait le modèle de la culture de convergence, qui proposait une forme de rapprochement interculturel axé sur la culture francophone comme point de ralliement », rapport Bouchard-Taylor (2008), p.117.

8. Ce débat civique-ethnique, où certains ont même opposé le civique et le culturel, est bien résumé par Bock-Côté (2007), dans son premier chapitre.

9. Selon Rosanvallon (1992, p.580), chercher à nier la nécessité de la catégorie de l'étranger pour définir une identité saperait toute possibilité de solidarité politique : «Cela reviendrait à vider de tout contenu l'idée de nationalité [ ] C'est nier du même coup l'existence d'une société politique distincte en son essence de la société civile, surtout si cette dernière est appréhendée sur un mode « différentialiste » (c'est-à-dire comme simple juxtaposition d'individus et de groupes hétérogènes). Plus aucun principe d'unité et d'identité collective ne peut alors être formulé : le social et le local deviennent des catégories terriblement abstraites, dont l'ouverture n'est paradoxalement plus du tout un gage d'insertion mais fonctionne au contraire comme une gigantesque machine à créer de l'indifférence entre les hommes, risquant par contrecoup de provoquer [des] crispation[s] identitaire[s]. » 
10. Ainsi, Josée Woerling soutient que l'article 43 de la Charte québécoise est l'équivalent de l'article 27 de la Charte canadienne, parce que la première doit être interprétée à la lumière de la seconde, et par exemple l'article 9.1 de la Charte québécoise est interprété à la manière de l'article 1 de la Charte canadienne, malgré des libellés très différents. L'auteur remercie Guillaume Rousseau pour ses précisions à ce sujet.

11. Par exemple, au sujet de cette compatibilité, Pierre-André Taguieff (2005) insiste sur la vitalité d'un communautarisme juif adhérant fortement aux idéaux de la République et à la laïcité française. Voir aussi Yves Lacoste (1997).

12. La Commission scolaire Marguerite-Bourgeois. Voir : http://www.radiocanada.ca/nouvelles/societe/2006/03/02/003-Kirpan-Coursup.shtml

13. http://www.radio-canada.ca/regions/Montreal/2008/10/29/002-kirpan-lasallevoies-fait.shtml

14. Les gains linguistiques de l'anglais et du français aux dépens des allophones étaient identiques à Montréal en 2001.

15. Pour ces chiffres, nous faisons références aux taux parmi les transferts linguistiques, et non aux taux de connaissance de la langue de la majorité. Ainsi, si 74\% des nouveaux arrivants allophones pouvaient converser en français au Québec en 2001 (Laura Julie-Perrault, « Francisation des immigrants - Les faits ", La Presse, 25 oct. 2007), 50\% des transferts linguistiques au Québec se font vers le français, et $50 \%$ vers l'anglais. Cf. Michel Paillé (2008). Le chiffre de 74\% n'induit donc pas une situation à celle qui prévaut au Canada anglais, puisque les transferts linguistiques au Canada anglais se font presque exclusivement vers l'anglais. Ils sont aussi supérieurs aux transferts linguistiques observés au Québec. À quoi il faut ajouter des transferts linguistiques de l'ordre de 30 à $40 \%$ des francophones hors Québec (de langue maternelle) vers l'anglais. En 1971, sur 2808000 allophones au Canada, 40000 parlaient français à la maison, et 1286000 parlaient anglais : Michel Paillé, Contribution à la démolinguistique du Québec, Conseil de la langue française, mai 1983 : http://www.cslf.gouv.qc.ca/publications/PubC141/ C141Tablc.html\#T-2

16. Rien n'interdit cependant de ramener le seuil de 50 à un plus bas niveau 20 par exemple.

17. «La nationalité suisse ne s'acquiert que par celui qui, après l'obtention de l'autorisation fédérale de naturalisation, est admis dans le droit de cité de la commune et du canton. En règle générale, la législation ne confère aucun droit à l'acquisition du droit de cité cantonal et communal. » Confédération suisse, office fédéral des migrations, « Naturalisation ordinaire » : http://www.bfm. admin.ch/bfm/fr/home/themen/buergerrecht/ einbuergerungen/ordentliche einbuergerung.html (Consulté le 23 févr. 2009).

\section{Bibliographie}

Accord Canada-Québec relatif à l'immigration et à l'admission des aubains, QUÉBEC, Ministère des Communautés culturelles et de l'immigration, 1991. http://www.micc.gouv.qc.ca/publications/fr divers/Accord-canada-quebecimmigration-francais.pdf

Charte de la langue française: http://www.olf. gouv.qc.ca/charte/charte/index.html. Commission scolaire Marguerite-Bourgeoys c. Multani, 4 mars 2004, jugement de la

Cour d'appel du Québec: http://www.jugements.qc.ca/php/decision.php?liste $=3547$ 
$7099 \& d o c=5206445857441 B 0 B \#$ ftnref2

Énoncé de politique en matière d'intégration et d'immigration, QUÉBEC, Ministère des Communautés culturelles et de l'immigration, 1990.

Multani c. Commission scolaire Marguerite-Bourgeoys, 2 mars 2006, jugement de la Cour suprême du Canada: http://csc.lexum.umontreal.ca/ fr/2006/2006csc6/2006csc6.html.

Affirmer les valeurs communes de la société québécoise, QUÉBEC, Ministère de l'immigration et des communautés culturelles, 2008. http://www.micc.gouv.qc.ca/ publications/fr/mesures/Mesures-ValeursCommunes-Brochure2008.pdf

Anctil, Pierre. «Défi et gestion de l'immigration internationale au Québec. » Cités, PUF. $\mathrm{n}^{\circ} 23$ (2005.3): 43-55: http://www.canada.uottawa.ca/images/docs/defi.et.gestionde. limmigration.internationale.au.quebec.pdf

Baillargeon, Stéphane. «Yolande James dévoile le plan d'intégration du gouvernement canadien - Les immigrants devront s'engager à respecter les valeurs québécoises », Le Devoir, 30 oct. 2008.

Bédard, Éric. « 1839, Rapport Durham: les écueils du progressisme. » dans C.-P. Courtois (dir.), "Anniversaires historiques », section de Miriam Fahmy (dir.), L'État du Québec 2009. Montréal: Fides, 2008: 478-481.

Bock-Côté, Mathieu. La dénationalisation tranquille. Montréal: Boréal, 2007.

Bouchard, Gérard et Charles Taylor. Fonder l'avenir. Le temps de la conciliation, rapport intégral, Commission de consultation sur les pratiques d'accommodement reliées aux différences culturelles, Québec, 2008.

Certeau, Michel de, Dominique Julia et Jacques Revel. Une politique de la langue: la Révolution française et les patois. L'enquête Grégoire. Paris: Gallimard, 1975.

Chevrier, Marc. « La petite politique d'un naufrage annoncé. » Dans « Le chaos universitaire », L'Action nationale (octobre 2008): 108-129.

- « Penser la res publica. Cet impensé qui nous gouverne. » L'Agora (mai-juin 1999): http://agora.qc.ca/textes/chevrier25.html.

Constant, Benjamin. De la liberté chez les Modernes. Éd. Marcel Gauchet. Paris:Hachette-Pluriel, 1980.

Corbeil, Jean-Claude. L'embarras des langues. Origine, conception et évolution de la politique linguistique québécoise, Montréal, Québec/Amérique, 2007.

Courtois, Charles-Philippe. Trois mouvements intellectuels québécois et leurs relations françaises: l'Action française, La Relève et La Nation, 1917-1939, thèse de doctorat en histoire, Institut d'Études politiques de Paris, 2007 et Université du Québec à Montréal, 2008, 2 vol.

- « Un rapport trudeauiste. » Dans « Bouchard-Taylor. Lectures malcommodes, III. » L'Action nationale (déc. 2008): 60-80.

- « Histoire, identité et démocratie. » Dans François Charbonneau et M. Nadeau (dir.). L'histoire à l'épreuve de la diversité culturelle. Bruxelles: Presses interuniversitaires-Peter Lang, coll. « Diversitas », 2008: 71-92.

- «Le républicanisme au Québec au début du XXe siècle: les cas de figure de Wilfrid Gascon, Olivar Asselin et Ève Circé-Côté. » Dans Marc Chevrier (dir.). L'idée de République au Québec, dans le Bulletin d'histoire politique 17. 3 (printemps 2009): 93-119.

Dubreuil, Benoît. «Intégration des immigrants - Pour une approche basée sur les résultats. » L'Action nationale (oct. 2006): 44-69. 
- « Pour bonifier la loi sur l'identité. » L'Action nationale (nov.-déc. 2007): 123-130.

- avec Dave Anctil: « La politique démocratique d'une société des identités: pour une défragmentation intellectuelle. » Jacques Beauchemin et Mathieu Bock-Côté (dir.), La Cité identitaire. Montréal: Athéna, 2007: 17-30.

Dufour, Christian. Les Québécois et l'anglais. Le retour du mouton. [s.1.]: Les éditeurs réunis (LÉR), 2008.

Dumont, Fernand. Le lieu de l'homme [1968]. Montréal: Bibliothèque québécoise, 2005.

Dutrisac, Robert. «Québec s'adresse en anglais aux trois quarts des allophones. » Le Devoir, 8 avril 2008.

- «Québec bafoue la Charte de la langue. L'État communique en anglais avec les entreprises établies au Québec. » Le Devoir, 17 avril 2008.

- « Jean Charest, redresseur de la loi 101? », Le Devoir, 24 et 25 octobre 2009.

Gervais, Lisa-Marie. « Premier colloque de l'Institut de recherche sur le français en Amérique - Québec devra prendre les grands moyens pour sauver le français dans la métropole ». Le Devoir, 29 nov. 2008.

Harvey, Louis-Georges. Le Printemps de l'Amérique française. Américanité, anticolonialisme et républicanisme dans le discours politique québécois, 1805 1837. Montréal: Boréal, 2005.

Kelly, Stéphane. La petite loterie. Ou comment la couronne a obtenu la collaboration du Canada français après 1837. Montréal: Boréal, 1997.

Kymlicka, Will. Liberalism, Community and Culture. Oxford University Press, 1989. - Multicultural Citizenship: A Liberal Theory of Minority Rights. Oxford University Press, 1995.

- trad. fr. La citoyenneté multiculturelle, Montréal-Paris, Boréal-

La Découverte, 2001.

Labelle, Micheline. 1996: «Immigration, politique du Québec », dans

L'Encyclopédie canadienne. http://www.thecanadianencyclopedia.com/index. $\mathrm{cfm}$ ?PgNm=TCE\&Params=F1SEC 860919

Lacoste, Yves. Vive la nation! Destin d'une idée géopolitique. Paris: Fayard, 1997.

Laforest, Guy. Pour la liberté d'une société distincte. Québec: PUL, 2004.

Lavergne, Armand. Trente ans de vie nationale. Montréal: Zodiaque, 1935.

Leroux, Georges. Éthique, culture religieuse, dialogue. Arguments pour un programme. Montréal: Fides, 2007.

Maclure, Jocelyn. « Les raisons de la laïcité ouverte. » Le Devoir, 24 nov. 2008.

Manent, Pierre. Histoire intellectuelle du libéralisme [1987].

Paris: Hachette Pluriel, 2002.

- Les Libéraux [1986]. Paris: Gallimard, 2001.

- La Raison des nations. Réflexions sur la démocratie en Europe. Paris: Gallimard, 2006.

Monière, Denis. Pour comprendre le nationalisme au Québec et ailleurs. Montréal: PUM, 2001. 
Paillé, Michel. Diagnostic démographique de l'état de la francisation au Québec, Commission de consultation sur les pratiques d'accommodement reliées aux différences culturelles, Québec, 2008, p.9.http://www.accommodements.qc.ca/ documentation/rapports/rapport-7-paille-michel.pdf

Paquette, Pierre. " Soustraire le Québec au multiculturalisme », Le Devoir, 9 mai 2008.

Parenteau, Danic. « La question nationale québécoise et le principe de la diversité culturelle. » L'Action nationale (nov. 2006): 46-52.

Pocock, John G.A. The Machiavellian Moment. Florentine Political Thought and the Atlantic Republican Tradition. Princeton: Princeton University Press, 1975. - trad.fr.: Le moment machiavélien, La pensée politique florentine et la tradition républicaine atlantique. Préface de J.-F. Spitz. Paris: PUF, 1997.

Quérin, Joëlle. " Georges Leroux, Éthique, culture religieuse, dialogue. Arguments pour un programme, Note critique. » L'Action nationale (février 2009): 94-101.

Rémond, René. L'invention de la laïcité française: de 1789 à demain. Paris: Fayard, 2005.

Renan, Ernest. Qu'est-ce qu'une nation? Paris: Calmann-Lévy, 1882.

Renaut, Alain. Libéralisme politique et pluralisme culturel. Nantes: Pleins Feux, 1999. Rosanvallon, Pierre. Le sacre du citoyen. Histoire du suffrage universel en France. Paris: Gallimard, 1992.

Rousseau, Guillaume. La nation à l'épreuve de l'immigration: les cas du Canada, du Québec et de la France, Éditions du Québécois, 2006.

Rousseau, Jean-Jacques. Du contrat social [1762]. Paris: Gallimard, 1964.

Schnapper, Dominique. La communauté des citoyens. Sur l'idée moderne de nation. Paris: Gallimard, 1994.

Sieyès, Emmanuel-Joseph. Qu'est-ce que le tiers-état? [s.1., 1789]. Éd. Jean-Denis Bredin. Paris: Flammarion, 1988.

Spitz, Jean-Fabien. Le moment républicain en France. Paris: Gallimard, 2005.

Taguieff, Pierre-André. La République enlisée. Pluralisme, communautarisme et citoyenneté. Paris: Syrtes, 2005.

Taras, Ray. Liberal and Illiberal Nationalisms. New York: Palgrave, 2002.

Thériault, Joseph-Yvon. Critique de l'américanité. Mémoire et démocratie au Québec. Montréal: Québec/Amérique, 2002.

Trudeau, Pierre Elliott. « La nouvelle trahison des clercs », Cité libre (avril 1962): 3-16.

Weinstock, Daniel. « Bouchard aurait dû s’y attendre », La Presse (11 juin 2008). 